\title{
Experimental study of liquid evaporation from a surface of gel mixture
}

\author{
Andrey Vyazmin ${ }^{1}$, Dmitry Khramtsov ${ }^{2 *}$, Boris Pokusaev ${ }^{2}$, Dmitry Nekrasov $^{2}$, \\ and Nikolay Zakharov ${ }^{2}$ \\ ${ }^{1}$ Moscow Technological University (MIREA), 119454 Moscow, Russia \\ ${ }^{2}$ Moscow Polytechnic University, Department of Chemistry, 107023 Moscow, Russia
}

\begin{abstract}
Experimental study of liquid evaporation from gel mixture surface was performed. Gels based on agarose and starch were studied. Dependencies of liquid evaporation from time were obtained for mixtures of agarose and starch. A non-linear dependency of evaporation speed was observed for all sets of mixtures. It was shown that presence of starch inhibits the process of liquid evaporation.
\end{abstract}

\section{Gel formation study}

The goal of studying gel formation was to identify intensity of liquid evaporation from a sample depending on gel and starch concentrations. This information can help to identify optimal time range when mixture is most suitable for injection from bioprinter. Such mixtures are served as bioink that forms structure for cells growth [1]. Making injection when gel is not formed enough leads to unsteadiness in a printed object, while keeping gel for too long make it loose flexibility which can lead to choking in a 3D bioprinter nozzle [2]. Living cells is a necessary component of bioink, hence it's important to study impact of living cells of gel formation. In a previous work authors studied living cells in gel using spectrometry analysis [3]. In this work an influence of living cells on liquid evaporation from a gel sample was studied.

\subsection{Experiment description}

Based on previous research, typical agarose mass concentrations were considered in the experiment -0.4 and $0.6 \%$. Additionally mixtures of agarose and starch were studied. Starch mass concentration varied from 0.1 to $0.3 \%$ for agarose-starch mixture preparation.

Experiments were performed using Petri dish and electronic scales for mass change fixation. Petri dish was isolated from electronic scales surface using foam plastic plate in order to ensure heat isolation of a Petri dish from scales. Hygrometer was used for humidity fixation and thermometer to ensure that all experiments were performed with the same temperature. In this experiment series temperature of $23^{\circ} \mathrm{C}$ and relative air humidity of $50 \%$.were maintained.

\footnotetext{
* Corresponding author: $\underline{\text { dp@ } @ \text { hramtsov.net }}$
} 
Additionally data about water evaporation in the same conditions was obtained. Evaporation from water free surface was used as a benchmark. For liquid evaporation numerical calculation was also performed. There are numerous approaches for calculating water evaporation from the surface, including empirical and based on diffusion equation. In this work evaporation was considered as occurring in isothermal conditions, not affected by external air movement, hence diffusion model of water evaporation into atmosphere from free surface can be applied.

\subsection{Evaporation model}

There is a wide range of models for calculation of evaporation process . In this study a liner model was applied [4]. Based on a Fick's law an evaporation flux was defined (1):

$$
F=-D \Delta C
$$

where $D$ is diffusion coefficient and $C$ - vapor concentration.

Assuming that initial mass of a sample in dimensionless units is 1 then change in mass of gel over the time due to evaporation can be described as following (2):

$$
m=1-(D \Delta C) \tau
$$

where $\mathrm{m}$ is a mass of a sample in dimensionless units at the time $\tau$, which is relative to experiment.

Comparison with experiment demonstrates that linear model of water evaporation doesn't describe precisely water evaporation in the experiment which can be related to influence of Petri dish borders and formation of micro dry spots due to thinness of water layer.

Obtained data shows that mixtures with starch demonstrate lower evaporation intensity and this intensity lowers with increasing starch concentration (fig. 1). Additionally all mixtures demonstrate the same behaviour in evaporation intensity with more intense evaporation during the first 10 minutes of experiment with consecutive flattening of mass reduction intensity.

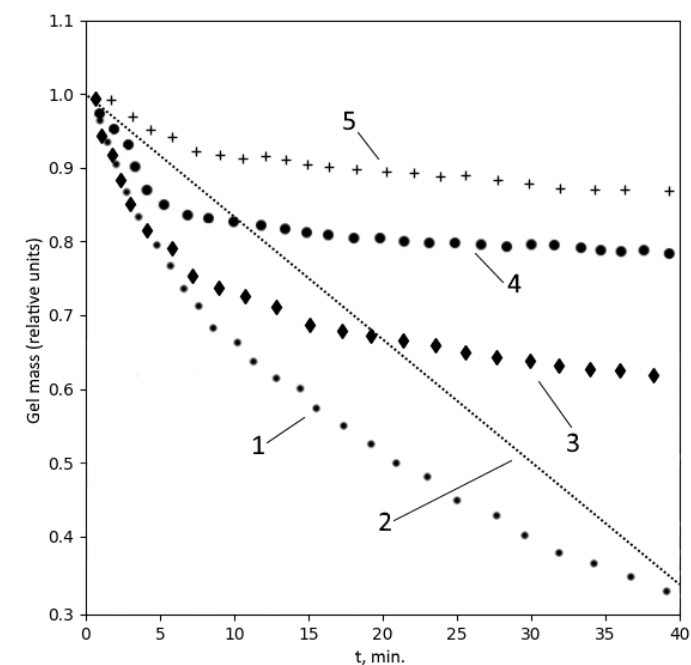

Fig. 1. Intensity of liquid evaporation during formation of agarose-starch samples. Water evaporation is used as a benchmark; 1 - water, experiment; 2 - water, model; 3 - agarose $0.4 \% ; 4$ - agarose $0.4 \%$, starch $0.1 \% ; 5$ - agarose $0.4 \%$, starch $0.3 \%$. 


\section{Analysis}

An additional experiment was set in order to explain rapid change in evaporation intensity after first 10 minutes.

\subsection{Evaporation dynamics}

A hypothesis stated that reduction in evaporation intensity is caused by a thin film of water that formed after sample is prepared. This liquid film causes more intensive evaporation in the beginning of the experiment and reduction happens after film is completely evaporated and further evaporation happens from the gel pores, hence the total area of evaporation reduces. To check this hypothesis a newly prepared sample of agarosestarch mixture was put into a thermostat that maintained the constant temperature of $35^{\circ} \mathrm{C}$ inside. This level of temperature allowed to keep sample in a liquid state for a longer time and hence such sample would maintain a liquid film on a surface for the whole period of experiment.

Experiment demonstrates that dynamics of evaporation of agarose-starch mixture in thermostat significantly differs from experiment in normal conditions (fig. 2). Moreover its intensity overpass evaporation intensity of pure gel sample in normal conditions. This data demonstrates that hypothesis of evaporation from a film located on an agarose-starch surface can be a promising concept that describes behavior of such mixture during liquid evaporation.

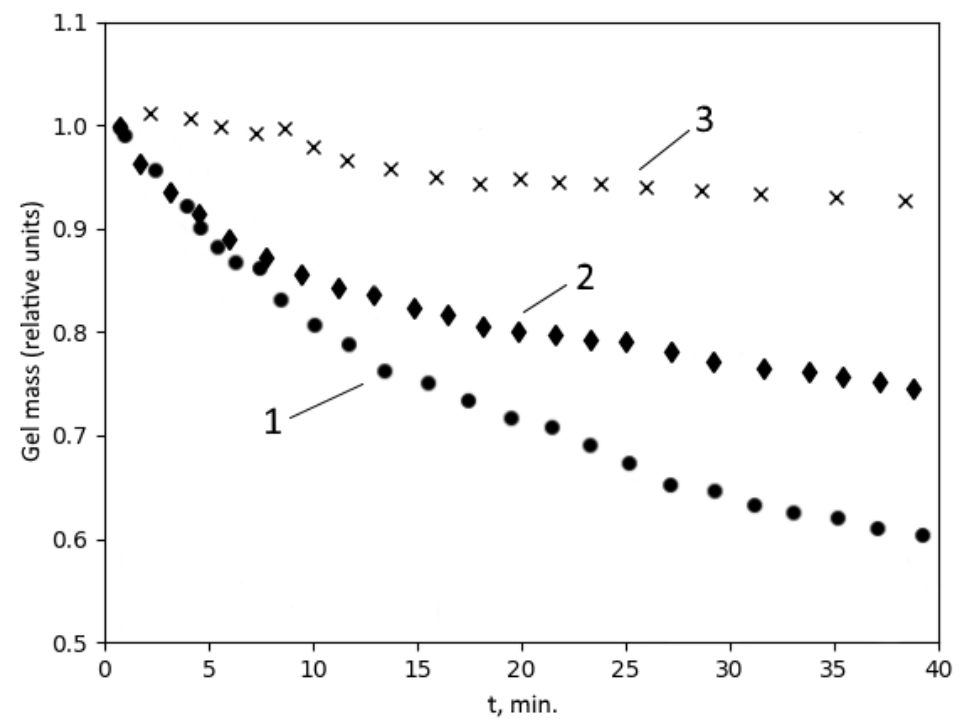

Fig 2. Intensity of liquid evaporation during formation of agarose-starch samples compared to the sample with maintained liquid layer on a surface. 1 - agarose $0.6 \%$, starch $0.2 \%$, temperature $35^{\circ} \mathrm{C} ; 2$ - agarose $0.6 \%$ normal conditions; 3 - agarose $0.6 \%$, starch $0.2 \%$ 


\subsection{Living cells}

Since the ultimate goal of the research is to find gel composition suitable for immobilization of living cells the next step was to find out the influence of cells on the process of liquid evaporation from agarose-starch samples. For this purpose yeast cells were used. A sample of yeast cells was added into a sample of gel. Yeast cells had 15\% mass concentration. Dependencies of liquid evaporation from agarose and agarose-starch samples with yeast cells were obtained and compared with corresponding samples measured without yeast cells (fig. 3).

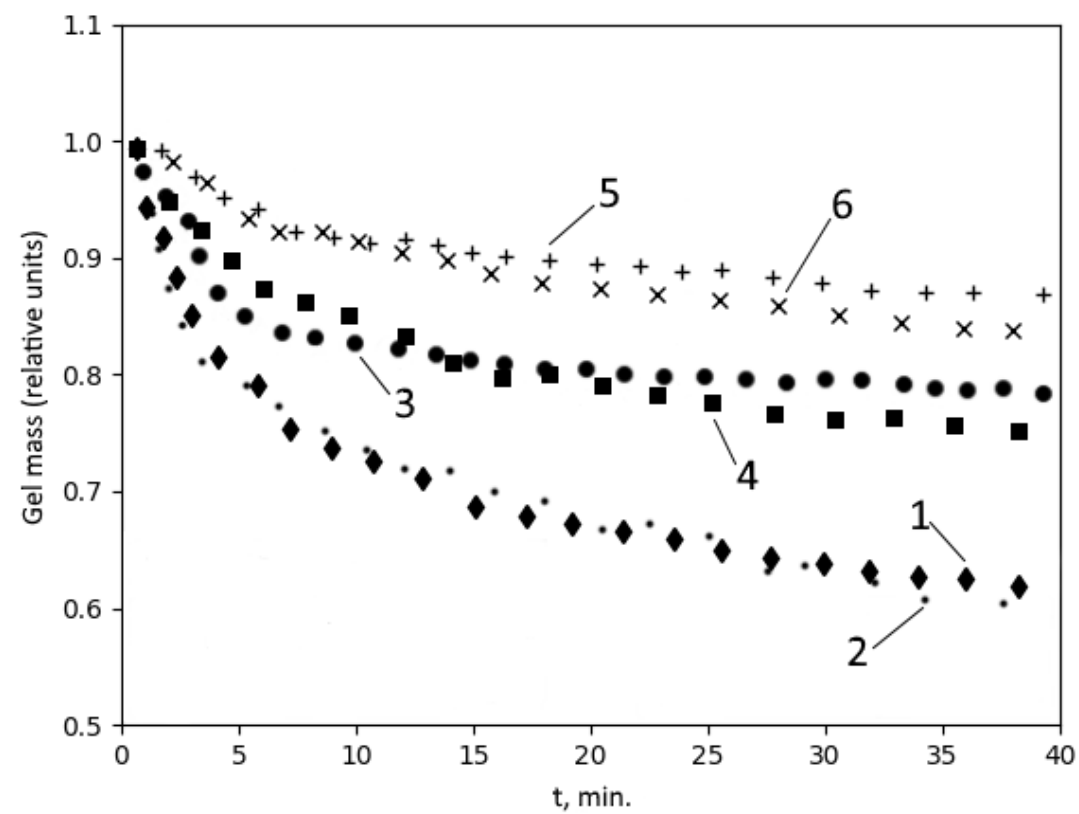

Fig 3. Intensity of liquid evaporation of agarose-starch samples with immobilized yeast cells. 1 agarose $0.4 \% ; 2$ - agarose $0.4 \%$ with yeast cells; 3 - agarose $0.4 \%$, starch $0.1 \% ; 4$ - agarose $0.4 \%$, starch $0.1 \%$ with yeast cells; 5 - agarose $0.4 \%$, starch $0.3 \% ; 6$ - agarose $0.4 \%$, starch $0.3 \%$ with yeast cells

\section{Conclusions}

Experimental study shows that dynamics of liquid evaporation demonstrates the same behavior in the initial evaporation phase and in case of liquid state of a gel which makes possible to admit that first phase of rapid evaporation is occurred due to a thin liquid film on a gel surface. Experimental data demonstrates that addition of yeast cells doesn't contribute to liquid evaporation intensity. This makes possible to conclude that the same time range of injection can be used for agarose-starch samples with and without yeast cells.

This work was supported by the Russian Science Foundation (project no. 15-19-00177). 


\section{References}

1. M. Kesti, M. Muller, J. Becher, M. Schnabelrauch, M. D’Este, D. Eglin, M. ZenobiWong, Acta Biomaterialia, A versatile bioink for three-dimensional printing of cellular scaffolds based on thermally and photo-triggered tandem gelation, 11, pp. 162-172 (2015)

2. I.T. Ozbolat, K.K. Moncal, H. Gudapati, Additive Manufacturing, Evaluation of bioprinter technologies, 13, pp. 179-200 (2017)

3. B. Pokusaev, S. Karlov, A. Vyazmin, D.Nekrasov, N.Zakharov, D.Khramtsov, D.Skladnev, D. Tyupa, JPCS, Structure of gels layers with cells, 925 (2017)

4. S.M. Rowan, M.I. Newton, G. McHale, J. Phys. Chem., Evaporation of Microdroplets and the Wetting of Solid Surfaces, 99, 13268-13271 (1995) 